\title{
Fusiones y adquisiciones: mecanismos de internacionalización de bancos latinoamericanos ${ }^{1}$ Mergers and acquisitions: Internationalization mechanisms of latin american banks
}

Recibido: 05 de abril de 2016 - Revisado: 14 de junio de 2016 - Aceptado: 29 de junio de 2016.

Julie Janeth Bertel Chadid ${ }^{2}$

José Antonio Uribe Herrera ${ }^{3}$

Sandra C. Gaitán Riaño ${ }^{4}$

Jaime Alberto Ospina Mejía ${ }^{5}$

\begin{abstract}
Resumen
Desde los años noventa, a nivel global, se han registrado un número elevado de fusiones y adquisiciones (en adelante $M \& A s$ ) en el mundo financiero cuyo objetivo es apalancar el crecimiento internacional del sector. Como la banca latinoamericana no ha sido la excepción, hemos examinado si las $M \& A s$ son mecanismos de internacionalización empleados por los bancos latinoamericanos; para ello, escogimos como casos de estudio el principal banco de Brasil, el de Colombia y el de Perú, observando el comportamiento del indicador y dimensionando el grado de internacionalización, durante el período 19902014. Aplicamos varias metodologías de medición a fin de no dejar por fuera aspectos importantes de la internacionalización. Los resultados del estudio presentan evidencia del uso de las $M \& A s$ por los bancos latinoamericanos como estrategias de internacionalización para llegar a nuevos mercados.
\end{abstract}

\section{Palabras clave}

Internacionalización, fusiones y adquisiciones, sector financiero, sector bancario.

\begin{abstract}
Since the 1990s, globally, there have been a large number of mergers and acquisitions (hereafter M \& As) in the financial world whose objective is to leverage the international growth of the sector. As Latin American banks have not been the exception, we have examined whether $\mathrm{M} \&$ As are internationalization mechanisms used by Latin American banks; For this, we chose as the case studies the main bank in Brazil, Colombia and Peru, observing the behavior of the indicator and dimensioning the degree of internationalization, during the period 1990-2014. We apply several measurement methodologies in order not to leave out important aspects of internationalization. The results of the study present evidence of the use of M \& As by Latin American banks as internationalization strategies to reach new markets.
\end{abstract}

Keywords

Internationalization, mergers and acquisitions, financial sector, banking sector.

\footnotetext{
${ }^{1}$ Artículo de investigación producto de trabajo de grado de la Maestría en Administración Financiera de la Universidad EAFIT, Medellín, Colombia. ${ }^{2}$ Magister en Administración Financiera, Universidad EAFIT, Medellín, Colombia.

Correo electrónico: jbchadid@gmail. com

${ }^{3}$ Magister en Administración Financiera, Universidad EAFIT, Medellín, Colombia.

Correo electrónico: jauribeh@hotmail. com

${ }^{4}$ Profesora Escuela de Economía y Finanzas, Universidad EAFIT, Medellín, Colombia. Correo electrónico: sgaitanr@eafit.edu.co

5 Profesor Escuela de Economía y Finanzas, Universidad EAFIT, Medellín, Colombia.

Correo electrónico: jospina3@eafit. edu.co

Para citar este artículo use: Bertel, J., Uribe, J., Gaitán, S., \& Ospina, J. (2017). Fusiones y adquisiciones: mecanismos de internacionalización de bancos latinoamericanos. Civilizar Ciencias Sociales y Humanas, 17(33), 135-148. doi: $10.22518 / 16578953.904$
} 


\section{Introducción}

En las últimas décadas se ha presentado una oleada de megafusiones y adquisiciones, producto de la globalización. Según Cardona Vélez (2014) esta tendencia se remonta al siglo XIX en Estados Unidos, en donde se formalizaron las primeras absorciones horizontales de empresas y que con el tiempo evolucionaron a integraciones verticales con el fin de fortalecer los conglomerados creados con anterioridad y responder al contexto económico de la época. Estas transacciones tuvieron una especial acogida después de la Segunda Guerra Mundial como estrategia de diversificación, en mayor medida en el sector bancario. Sin embargo, a partir de la última década del Siglo XX se presentó una oleada de transformación industrial como mecanismo eficiente frente a la apertura de servicios, la desregularización de los mercados y los desarrollos tecnológicos, lo que dio origen al auge de las grandes transacciones de $M \& A s$.

Las estrategias antes mencionadas han sido utilizadas para incursionar, crecer y consolidarse fuera de los países de origen, proceso que se conoce como internacionalización. Dicho proceso, según Johanson y Vahlne (1997), obedece a una serie de decisiones corporativas que tienen en cuenta la previa experiencia o el conocimiento para identificar los lugares y la metodología de crecimiento en otros países, la distancia psicológica (se le denomina así a la distancia cultural que existe entre un país y otro), y cuyos pasos se dan de forma gradual. Por otra parte, Forsgren y Johanson (1992) la definen como una reacción ante cambios estratégicos del mercado que, por supervivencia y crecimiento, impulsan a las empresas a ingresar a nuevos territorios. Adicionalmente, es importante señalar que la estrategia de internacionalización requiere alta flexibilidad para responder a las oportunidades y amenazas, al igual que para identificar su ventaja más fuerte y seleccionar el país que beneficie la operación futura (Vermeulen, 2001).

Aunque muchos autores hablan del proceso de internacionalización de las empresas, escasos son los que se han referido al grado de internacionalización (medición de un conjunto de indicadores); A falta de medidas confiables para establecer el grado de internacionalización que se da como causa de las investigaciones empíricas que se realizan para establecer la validez de las mediciones, las cuales no cuentan con la robustez necesaria para lograr un análisis completo de variables, conceptos y contexto de la información utilizada para dichos estudios (Sullivan, 1994).

Encontramos trabajos que han observado las causas de éxito y de fracaso en casos empresariales de internacionalización: Vermeulen (2001) estudió a Randstad, una empresa de trabajo temporal de los Países Bajos que, con enfoque, paciencia y con una alta inversión económica y gerencial en la adquisición de empresas, logró aumentar cinco veces el precio de su acción en cuestión de cinco años.

Como mencionábamos al inicio del artículo, las actividades comerciales e inversiones bancarias internacionales han tomado un gran impulso desde los años noventa. A partir de esta época, la inversión de bancos extranjeros en países en desarrollo se ha incrementado, pasando de US\$545,200 millones en 1985 a US\$1,318.8 millones en el 2000 (Martinez, Powell, \& Vladkova-Hollar, 2005). El sector financiero ha utilizado las fusiones y adquisiciones internacionales como una alternativa para contrarrestar las crisis económicas, producto de la débil estructura del sector y la disminución del ahorro. Es así como las entidades bancarias buscan implantarse fuera de sus países de origen, llegar a nuevos mercados y diversificar el portafolio de productos, para garantizar la generación de valor a sus accionistas. Varios trabajos de literatura económica plantean que las $M \& A s$ se llevan a cabo, entre otros motivos, para mejorar la rentabilidad, la eficiencia y las formas de negociación.

Con nuestro trabajo analítico, buscamos evaluar si las $M \& A s$ son un mecanismo para 
la internacionalización, entendida como una forma de tener brazos comerciales o productivos extendidos en el exterior (ProMéxico, s.f.).

Para ello, tomamos como casos de estudio los bancos que ocupan el primer lugar (definido así en términos de activos) en los países con información disponible durante el período 1990-2014, los cuales son: Banco do Brasil (Brasil), Bancolombia (Colombia) y Banco de Crédito del Perú (Perú).

Hemos empleado tres medidas de grado de internacionalización: a partir de los activos, de la utilidad y de los ingresos (Daniels \& Bracker, 1989; Stopford \& Wells, 1972; Vernon, 1971).

El análisis muestra que no es recomendable basarse en una sola medición debido a que se pueden omitir características importantes que afectan esta medida. Adicionalmente, aunque las $M \& A s$ son mecanismos para incursionar en los mercados internacionales, las mediciones utilizadas se pueden ver afectadas por la consolidación del mercado local del banco.

\section{Marco conceptual}

Las finanzas corporativas se enfocan en las decisiones financieras que toman las empresas y el estudio de su impacto en la generación de valor (Ross, Westerfield, \& Jaffe, 2014). Sumado a esto, Mascareña (1999) se refiere a las fusiones y adquisiciones como mecanismos que permiten el uso eficiente de los recursos de la empresa.

Una fusión es una operación utilizada para unificar inversiones y criterios comerciales de dos compañías en una sola (Cisneros \& Jiménez, 2014; Santeiro, 2014). Por su parte, la adquisición es el proceso directo realizado por una empresa para tomar el control operacional de otra bien sea por compra directa o acumulación de acciones (Cardona-Vélez, 2014; Cisneros \& Jiménez, 2014). En una empresa, ambas operaciones se realizan con el propósito de ser más eficiente en la gestión de costos, aportar mejoras de insumos o productos, $\mathrm{u}$ otorgar poder de mercado que le permita implementar una estrategia de fijación de precios (Estrada, 2014). Además, pueden utilizarse como alternativa para replantear los negocios y así mitigar el riesgo, en cuanto a inversión y tiempo, para crear una empresa completamente nueva (McCann \& Gilkey, 1990).

Son diversas las razones que llevan a efectuar $M \& A s$, entre otras, encontramos: la generación de sinergias operativas donde se espera lograr economías de escala o de alcance; sinergias financieras para disminuir costo de capital; diversificar productos, canales o mercados; realineamiento estratégico que permita adquirir capacidades para adaptarse con mayor rapidez a las exigencias del mercado; la empresa adquirente confía en que la valoración de la empresa objetivo es más acertada que la del mercado; comprar activos subvalorados; estrategia impositiva donde la empresa que adquiere o fusiona obtiene ventajas económicas por efectos impositivos, $\mathrm{y}$ por último, porque el poder de mercado es un atractivo para la empresa adquirente ya que al incrementar la participación de mercado mejora la habilidad de fijar los precios por encima de los niveles competitivos (DePamphilis, 2012).

\section{Concepto de internacionalización.}

De acuerdo con la Comisión Económica para América Latina y el Caribe -Cepal(2011), en los años noventa se evidenció la necesidad de "supervivencia" de la banca en un mercado globalizado y de alto desarrollo tecnológico, lo cual forzó a las empresas del sector financiero a una rápida e intensa integración de los mercados para aprovechar la liberación de los movimientos de capital en la mayoría de economías del mundo. Esta tendencia surgió principalmente en países desarrollados y luego se extendió por América Latina. 
La internacionalización es un proceso y se considera que es parte de los efectos de la globalización; esta a su vez, se comprende como fenómeno económico producto del desarrollo tecnológico, los avances de las comunicaciones y la reducción de costos de transporte, que permite la eliminación de las fronteras entre países y convierte al ser humano en un ciudadano del mundo (Torres, 2014). Este fenómeno promovió en el sector financiero la búsqueda de oportunidades de negocio en mercados extranjeros dado el evidente crecimiento superior de la actividad bancaria internacional frente al comercio mundial de bienes y por la eliminación de los controles de capital en la mayoría de los países industrializados, para así aprovechar a su favor la disminución de la carga regulatoria, en un contexto divergente entre los países (Larraín, 1995). A eso se suma que suscitó utilizar el conjunto de productos, clientes y ubicación geográfica como medio para diversificar los ingresos (Rodrigues de Paula, 2003), y de manera sistemática para que la experiencia sea un impulsador de la internacionalización (Vermeulen, 2001).
Las transacciones $M \& A s$ han sido un mecanismo para desarrollar las estrategias de internacionalización del sector bancario. Esto se observa al analizar las transacciones de la banca mundial que entre los años 1985 y 2001 participaron del $20 \%$ de las operaciones. Si se compara con otras industrias como la manufacturera, vemos que sus operaciones apenas representaron el $34 \%$ en 1990, mientras que el sector financiero llegó a $13 \%$ y el promedio de todas las industrias fue del $24 \%$ (Buch \& DeLong, 2003). Se evidencia, entonces, que en los últimos años los jugadores más importantes de la industria comienzan a abrirse terreno en ámbitos internacionales y los negocios de $M \& A s$ transnacionales aumentan su intervención.

El sector financiero latinoamericano se ha valido, durante las últimas tres décadas, de las $M \& A s$ internacionales como estrategia para propiciar el crecimiento y fortalecimiento de la industria. En la gráfica 1 se observa el comportamiento y la evolución de $M \& A s$, domésticas e internacionales, en Argentina, Brasil, Chile, Colombia, México y Perú.

Gráfica 1. Número de transacciones de $M \& A s$

Argentina

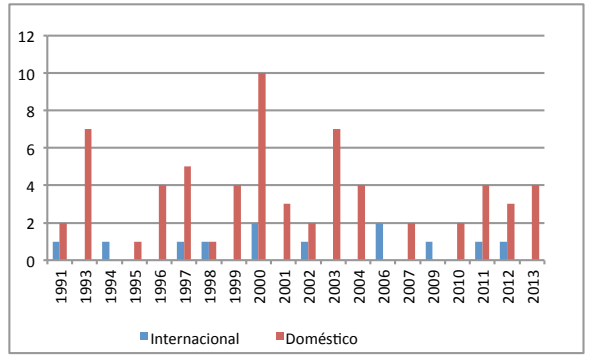

Chile

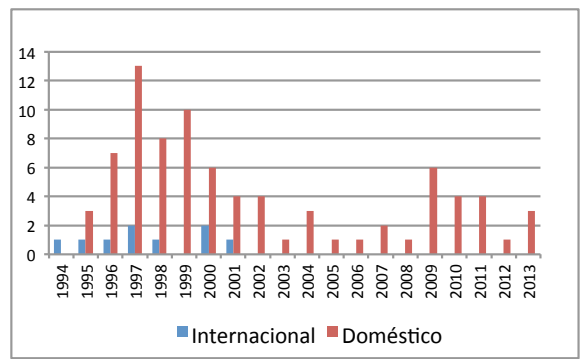

Brasil

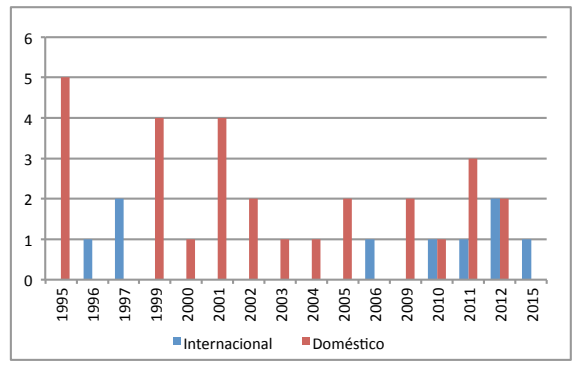

Colombia

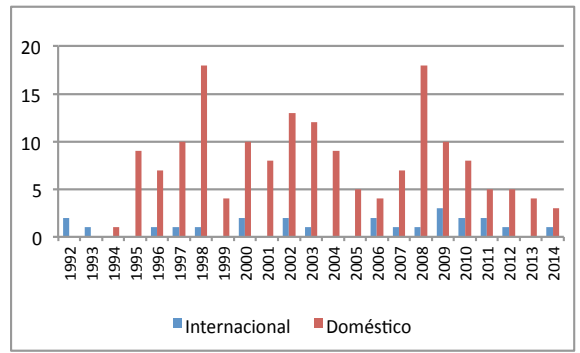


México

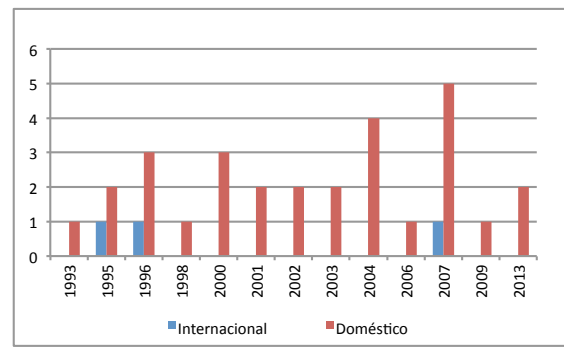

Perú

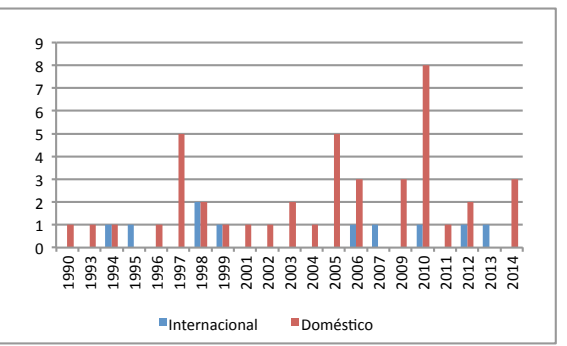

Fuente: elaboración de los autores con base en Thomnson One, 2015.

Sibien labancamundialsehacaracterizado por movimientos importantes de este tipo de transacciones, en los países latinoamericanos se evidencia un comportamiento diferente en cuanto a la evolución de las estrategias de $M \& A s$; de ello se puede concluir que no es una estrategia regional, sino el reflejo de las circunstancias de cada país y de las necesidades de crecimiento de los mismos.

Argentina y Brasil podrían considerarse los pioneros en la región: Argentina muestra una fuerte concentración, de 1995 a 2001, del mayor volumen de transacciones domésticas $\mathrm{y}$, en lo primordial, de las internacionales, que son las que se mantienen en el tiempo pero con menor intensidad. De los años recientes no hay informes de transacciones domésticas, lo que describe que su sector financiero tiene una clara tendencia hacia la estrategia de internacionalización.

Brasil, el país latinoamericano con mayor volumen de transacciones internacionales desde 1995, refleja un comportamiento cíclico. Sin embargo, durante los últimos cinco años la tendencia ha sido negativa dado que las transacciones domésticas se muestran estables a lo largo de los años, sin presentar algún repunte que manifieste una variación importante de la economía brasileña.

En cuanto a Colombia y Perú, encontramos que durante algunos años presentan altos repuntes de transacciones internacionales. Si se descartan esos períodos, observamos que el comportamiento es estable o similar a lo largo del tiempo; incluso, en algunos años son parecidos los compartimientos de las $M \& A s$ internacionales con los de las nacionales. El sector financiero de Colombia refleja una estrategia local e internacional pues no se han dejado de realizar transacciones de las dos tipologías; en este punto coincide con México, pero difiere con Chile y Perú, países en los que durante períodos largos, únicamente se realizan transacciones internacionales.

Las fusiones y adquisiciones (tanto en transacciones internacionales como en domésticas) pueden tener motivaciones afines; a pesar de ello queremos señalar que, variables como el costo de la información, producto de la distancia; el lenguaje: la cultura; la moneda; la regulación económica y financiera del país; así como la situación macroeconómica de los otros países (Buch \& DeLong, 2003), son aspectos importantes a considerar en las operaciones de $M \& A s$ transnacionales.

Se pensaría que la diversificación geográfica también puede ser una de las razones de dichas operaciones; no obstante, hay estudios que tratan de demostrar que firmas con mayor alcance geográfico y con economías de escala no necesariamente tienen un menor riesgo que compañías pequeñas totalmente especializadas (De Nicoló, Bartholomew, Zaman y Zephirin, 2004).

Pocos autores tratan el tema de las metodologías de medición del grado de internacionalización de las empresas; y mucho menos, 
específicamente en el sector financiero. Para conocer qué tan internacionalizados están los jugadores de la banca latinoamericana, es importante entender cómo se estima el grado de internacionalización que permite evidenciar la tendencia que presentan las transacciones de $M \& A s$ transnacionales.

Ren, Eisingerich y Tsai (2014) sugieren que dicha medición se puede establecer como la relación entre las ventas por exportaciones y los ingresos totales de ventas. Por su parte, Sullivan (1994) afirma que la estimación de este indicador es arbitraria, teniendo en cuenta que no ha sido posible crear una metodología de verificación que abarque los conceptos teóricos y los resultados empíricos. Sullivan (1994), lo estima como la relación entre ventas internacionales y ventas totales; otra forma es el cociente entre activos internacionales y totales de la empresa; por último, la relación que hay entre el número de oficinas en el exterior y el total de las sucursales o filiales (Stopford \& Wells, 1972; Vernon, 1971). Por otra parte, la Asociación gremial financiera colombiana -Asobancaria- (2013) determina el grado de internacionalización midiendo la participación de la inversión local frente a la extranjera, y estima la participación de la banca local en el exterior utilizando el crecimiento de oficinas subordinadas de bancos colombianos en el exterior en un período de tres años.

\section{Metodología}

La base de datos consultada para el desarrollo de la investigación es la Thomnson One (2015); de ella se extrajo la información relacionada con las transacciones de $M \& A s$ internacionales de bancos comerciales latinoamericanos, del período comprendido entre 1990 y 2014.

Se pretendía estudiar el primer banco de todos los países de Latinoamérica y determinar para cada uno de ellos el grado de internacionalización a partir de las $M \& A s$; sin embargo, solo fue posible obtener la información financiera del Banco do Brasil (Brasil), de Bancolombia
(Colombia) y del Banco de Crédito del Perú (Perú). Estos bancos ocupan el primer lugar por nivel de activos y son el objeto de estudio del presente trabajo.

En primer lugar, y recuperando información de Thomnson One (2015), se estableció la cronología de las transacciones (domésticas e internacionales) con especial atención a las que realizaron fuera de sus fronteras. A continuación, se consultaron en S\&P Capital IQ - McGraw Hill Financial (2015) los estados financieros históricos anuales de los bancos (adquiridos y adquirentes) en dólares; y se aplicó la tasa de cambio representativa del mercado (TRM) de cada país al 31 de diciembre de cada año.

Para cada transacción internacional identificada, se procedió a obtener los valores de utilidad neta, activos totales y ventas; después se determinó el grado de internacionalización de la siguiente manera:

A partir de los activos: se analiza cuál fue el crecimiento o la disminución del cambio del indicador de los activos en el período anterior y posterior a la transacción (Stopford \& Wells, 1972; Vernon, 1971). A continuación, el grado de internacionalización se estima como la variación (crecimiento o decrecimiento) de la razón entre los activos en el exterior, con respecto al total de los activos del banco fusionado.

A partir de la utilidad: se calcula como la variación (crecimiento o decremento) de la razón entre la utilidad en el exterior, con respecto al total de la utilidad del banco fusionado; esta medición permite entender cuál fue el crecimiento o decremento del indicador de la utilidad en el período anterior y posterior a la transacción.

A partir de las ventas: se establece el crecimiento o decremento del indicador de ventas en el período anterior y posterior a la transacción (Daniels \& Bracker, 1989). Se estima como la variación (crecimiento o decremento) de la razón 
de ventas en el exterior, con respecto al total de las ventas totales del banco fusionado.

Al llegar a este punto y para terminar, aplicamos un análisis comparativo del comportamiento y la tendencia del grado de internacionalización entre los diferentes bancos.

\section{Resultados}

\section{Bancolombia.}

Compañía declarada como grupo financiero latinoamericano, con presencia en Colombia, Costa Rica, El Salvador, Islas Caimán, Guatemala, Panamá, Perú y Puerto Rico. La organización ha utilizado las $M \& A s$ para consolidarse local y regionalmente y esto se evidencia en su historia: Bancolombia nació de la fusión, en 1998, del Banco Industrial Colombiano y del Banco de Colombia; en 2005, se realizó la fusión entre Bancolombia, Conavi y Corfinsura. El paso a las transacciones internacionales se inició con la compra, en 2006, de Banagrícola en El Salvador; en 2007 adquiere el 40\% del grupo Agromercantil de Guatemala; y, en 2013, compra el $90 \%$ de la operación de HSBC Panamá. Con todas estas operaciones (adquirió el $100 \%$ de cada una de las empresas por medio de transacciones de compra y venta), Bancolombia se ha consolidado como grupo financiero regional (Grupo Bancolombia, s.f.).

En la gráfica 2, se presenta la estimación del grado de internacionalización desde el punto de vista de las $M \& A s$, medido a partir de los activos, utilidad e ingresos. El indicador de internacionalización comienza en 2006.

Gráfica 2. Grado de internacionalización de Bancolombia.

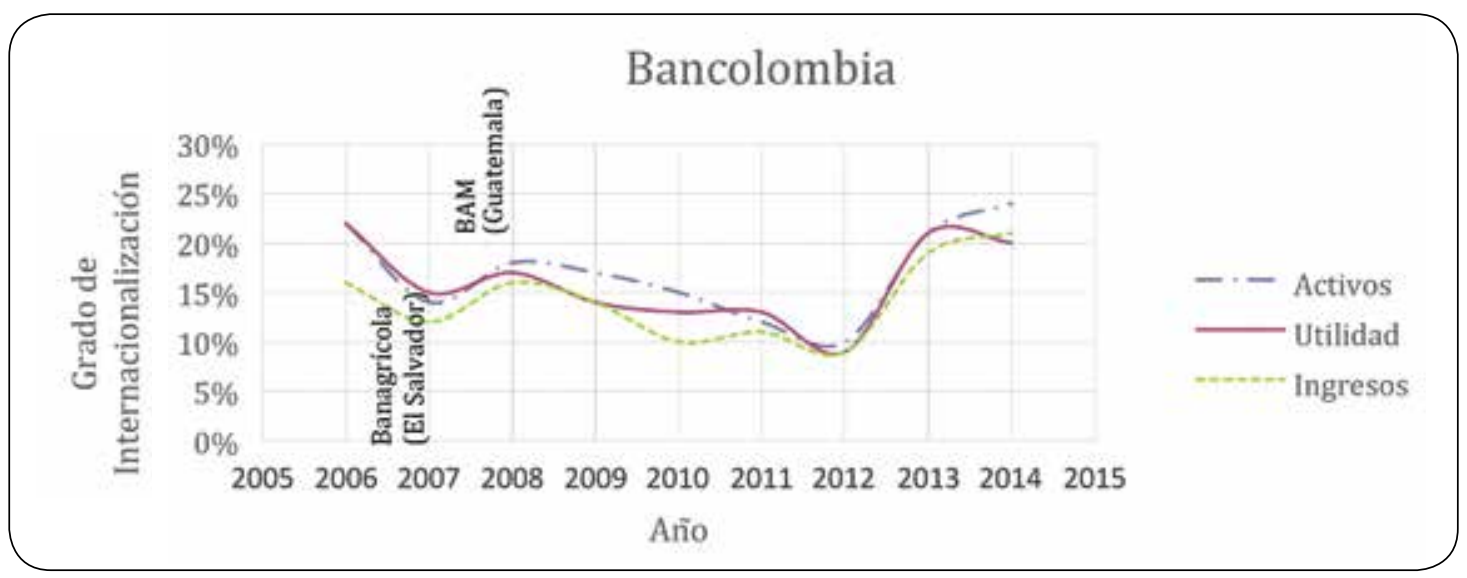

Fuente: elaboración de los autores con base en información de IQ McGraw Hill Financial, 2015 y tasa de cambio representativa del mercado (TRM) histórica, Investing, 2015.

Con base en la ilustración, se identificó que los negocios en el extranjero, en conjunto, son más eficientes que el negocio local, debido a que el grado de internacionalización por ingresos resulta ser menor que el grado por activos. Aun así, el grado determinado de acuerdo con la utilidad no cae y se mantiene cerca del calculado por activos. Bancolombia, con menores ingresos, reporta una utilidad similar en términos relativos al tamaño de los activos. Al mismo tiempo, la rentabilidad sobre los activos de los negocios locales e internacionales es similar, dado que los grados de internacionalización por activos y por utilidad son muy cercanos.

En cuanto a la evolución del indicador de internacionalización, se aprecia que en 2006 comenzó con $22 \%$ a partir de activos y de utilidad, y de $16 \%$ según ingresos. Sin embargo, para el año siguiente decreció $36 \%$ 
frente a un crecimiento de los activos totales de $67 \%$; por lo tanto, se puede concluir que se presenta un fortalecimiento del negocio local. Para los años 2013 y 2014, los resultados son comparables al de 2006, lo que permite argumentar un mayor crecimiento del negocio interno: con solo tres adquisiciones locales, se obtuvo el mismo grado de internacionalización que alcanzó en 2006 con una sola adquisición internacional.

\section{Banco do Brasil.}

Empresa constituida con capital privado y del Estado, enfocada en la atención a los diferentes segmentos personales y a empresas pequeñas y grandes (Banco do Brasil, s.f.). En su historia se percibe una marcada consolidación dentro de las fronteras de Brasil.

En la gráfica 3 se observa cómo el grado de internacionalización de este banco comienza a evolucionar a partir del año 2009 con la adquisición parcial del Banco Patagonia. Se puede apreciar cómo el grado de internacionalización por activos representa, en el año 2014 , tan solo un $0.5 \%$ del tamaño del negocio, sin embargo, se evidencia cómo el negocio extranjero obtuvo una rentabilidad sobre el activo mayor, debido a que el grado de internacionalización por utilidades es mayor que el del mismo tipo por activos.

\section{Gráfica 3. Grado de internacionalización del Banco do Brasil.}

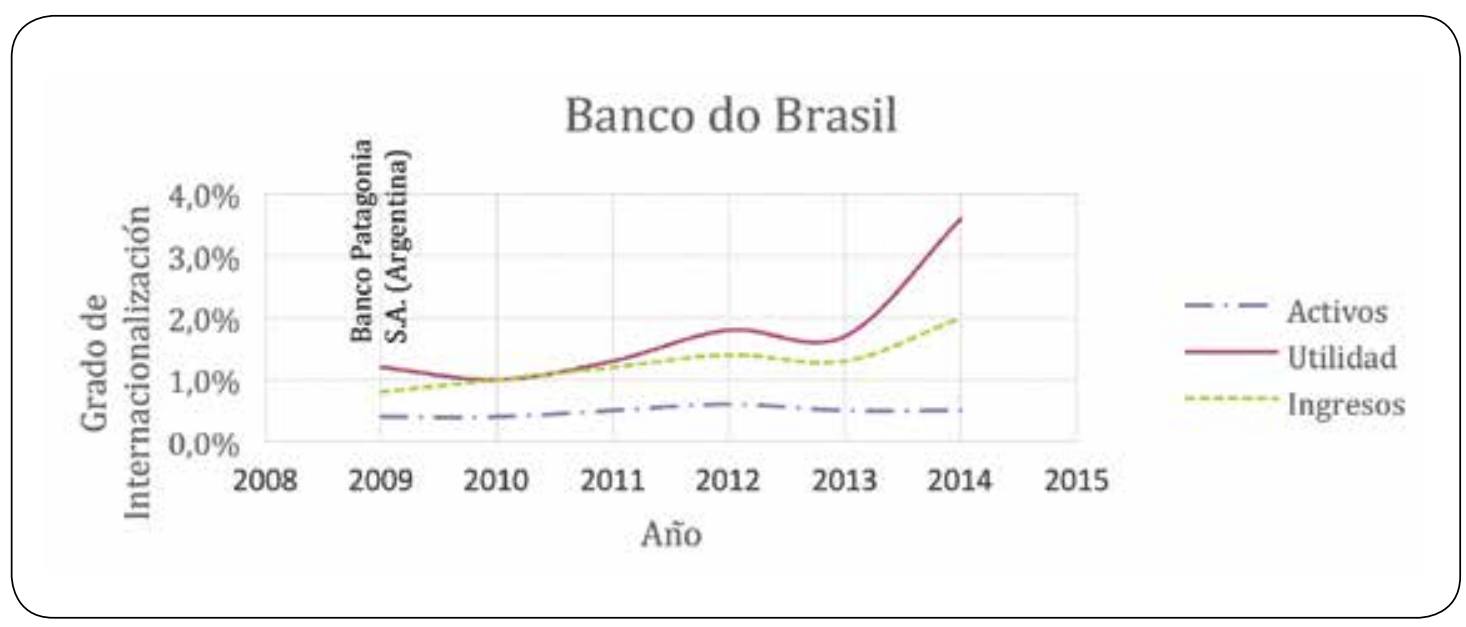

Fuente: elaboración de los autores con base en información de IQ McGraw Hill Financial, 2015 y tasa de cambio representativa del mercado (TRM) histórica, Investing, 2015.

En el 2014, su negocio internacional (obtenido mediante transacciones de $M \& A s$ ), representa el $0,53 \%$ del total de su negocio; el $99,5 \%$ es un negocio doméstico, pero el $3,6 \%$ de su utilidad proviene del negocio internacional, lo que, en comparación, indica que el negocio internacional tiene un retorno sobre activos (ROA) casi ocho veces mayor que el del negocio doméstico: por tamaño de activos da un $0,53 \%$ y por utilidad un $3,6 \%$. Se observa que el $2 \%$ del total de sus ingresos son internacionales, es decir, que si se compara con el tamaño de activos, se constata que los ingresos del negocio internacional son mayores, en términos relativos, frente al activo; comparándolo con la utilidad muestra que el negocio internacional es más eficiente porque logra pasar del $2 \%$ al $4 \%$. Resumiendo, una vez comparadas la rentabilidad y la eficiencia en las $M \& A s$ locales y en el extranjero, se constata que son más eficientes los recursos invertidos en las operaciones internacionales. 


\section{Banco de Crédito del Perú (BCP).}

Sociedad peruana que inició sus actividades en 1889 con el nombre de Banco Italiano. Tiene una clara orientación a fortalecer su pre- sencia internacional: es el único banco peruano que cuenta con oficinas directas en Nassau y Nueva York, y adelantó la compra parcial del Banco de Crédito e Inversiones (BCI) de Chile (Thomnson One, 2015).

Gráfica 4. Grado de internacionalización del Banco de Crédito del Perú (BCP).

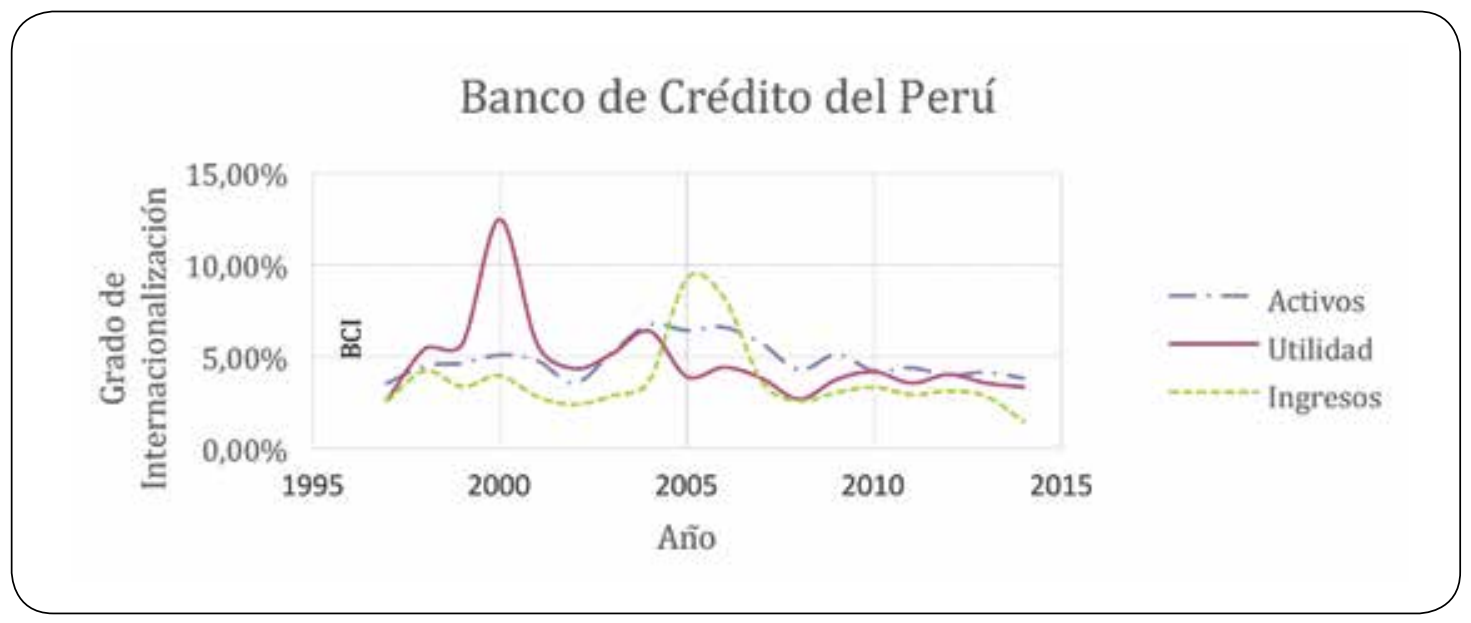

Fuente: elaboración de los autores con base en información de IQ McGraw Hill Financial y tasa de cambio representativa del mercado (TRM) histórica, Investing, 2015.

El grado de internacionalización del Banco de Crédito del Perú se activa en 1997 con la compra parcial del BCI en Chile, pero hasta 2014 ha sido su única experiencia de $M \& A s$ fuera de sus fronteras. En cuanto al valor de sus activos, se registra que el BCP y el BCI son muy similares en tamaño; también se observa que el crecimiento del BCI, desde 1997 hasta 2007, fue mayor con respecto al crecimiento del $\mathrm{BCP}$, lo cual generó una tendencia creciente (hasta 2007), en el grado de internacionalización. A partir de ese año se reconoce, en el valor de sus activos, que el Banco de Crédito del Perú comenzó a crecer a una mayor rata que el BCI, lo que generó que, para 2014, su grado de internacionalización se acercase a los niveles iniciales de 1997.

Aquí conviene detenerse un momento a fin de señalar que, para el Banco de Crédito del Perú, la inversión realizada en el BCI fue más una decisión puntual $\mathrm{y}$, posiblemente, estratégica. Eventualmente, no se adoptó como un objetivo de internacionalización, si se tiene en cuenta que durante diecisiete años no ha realizado otro compromiso internacional y que, por el contrario, ha logrado una consolidación local en los últimos siete años.

\section{Análisis entre bancos.}

Cada uno de los bancos se encuentra en situaciones muy diferentes de internacionalización, aun siendo los pioneros en sus respectivos países. Banco do Brasil, a pesar de ser el jugador más grande en tamaño de activos (casi diez veces más que Bancolombia), presenta un indicador de internacionalización aproximadamente 6 a 20 veces por debajo, según el indicador que se estudie. Lo anterior pone en evidencia que el tamaño de los mercados locales es muy importante: Banco do Brasil puede ser diez veces más grande (que Bancolombia) manteniendo un epicentro muy local y habiendo realizado hasta hace muy poco tiempo (2009), su primera transacción de $M \& A s$ internacional. Si se con- 
sideraran de manera estricta los resultados obtenidos en los grados de internacionalización, parecería una adquisición pequeña, y en términos relativos, por supuesto, puede serlo, pero hay que señalar que la adquisición parcial del Banco de Patagonia es casi de la misma importancia que la de Banagrícola para Bancolombia.

Con base en lo anterior, se puede afirmar que el grado de internacionalización es una medida concerniente a cada "jugador" y que sirve para identificar cómo está posicionado a nivel internacional; aun así, no debería pensarse que una compañía que presenta un alto grado de internacionalización, necesariamente realiza negocios de mayor importancia versus otra empresa con menor grado de internacionalización, porque, según el tamaño local de cada jugador, el grado de internacionalización se ve afectado; no obstante, sí es factible comparar qué tan diversificado tiene el negocio, en el sentido geográfico, una compañía respecto a otra.

Sumado a lo anterior y según se observa en las gráficas 2, 3, y 4, un aumento en el grado de internacionalización no siempre responde al hecho de adquirir un banco en un país extranjero o por un crecimiento en las variables (activos, utilidades o ingresos en el exterior) involucradas en su estimación, ya que dependiendo de la variable utilizada, puede ser una situación local lo que afecta el cálculo. Es el caso de Bancolombia, para el cual, en 2007 y a pesar de la adquisición de BAM en Guatemala, el indicador de grado de internacionalización (utilizando las tres medidas propuestas) cae debido a su consolidación en el mercado local (Colombia); esta situación se repite en el año 2012, ya que el crecimiento local es mayor que el internacional. En todo caso, en el 2013, la compra del HSBC Bank en Panamá es un factor importante que se refleja en el aumento del indicador.

En la gráfica 3 se observa cómo el Banco Do Brasil mantiene el indicador del grado de internacionalización, en cuanto a activos se refiere, muy constante, y esto indica que a nivel de activos no realizó nuevas adquisiciones; sin embargo, erróneamente se podría concluir que el rendimiento, en términos de utilidad e ingresos, fue creciente; lo anterior no es cierto ya que este aumento es explicado por la disminución en las utilidades e ingresos del mercado local.

Situación parecida presenta el Banco de Crédito del Perú en el año 2000, donde el aumento en el grado de internacionalización, medido a partir de las utilidades, responde a una caída en el mercado local. Situación opuesta se le presenta en 2005: el indicador crece de forma abrupta (basados en la medida de los ingresos), por un aumento de los ingresos en el mercado internacional, mientras que la media por utilidades muestra un desplome.

En suma, y continuando con la comparación entre las tres firmas estudiadas, se puede afirmar que Bancolombia es el banco más diversificado, desde el punto de vista geográfico, con respecto a su lugar de origen, así como el que ha venido haciendo más transacciones de $M \& A s$ internacionales. Lo anterior lleva a considerar que, cuando se contrasta el grado de internacionalización de empresas originarias de distintos países, no necesariamente la que esté más diversificada tendrá un menor riesgo, pues se están comparando indicadores y geografías de países diferentes, lo que supone niveles de riesgo dispares. Por tanto, esta comparación podría tener más sentido cuando se realiza entre compañías de un solo país.

\section{Conclusiones}

A partir de la cantidad importante de transacciones $M \& A s$ de tres de los principales bancos latinoamericanos, este estudio presenta la estimación del grado de internacionalización de los mismos, medido con base en su nivel de activos.

No existe un único argumento para afirmar si el indicador de internacionalización 
traduce algo positivo o negativo; este puede arrojar un valor según los resultados del negocio local o teniendo en cuenta la finalidad por la cual se haya realizado la operación de $M \& A s$. Por ejemplo, Bancolombia refleja una estrategia clara de internacionalización, pero sigue fortaleciendo a un mayor ritmo el negocio local, lo que disminuye el grado de internacionalización.

Con el indicador de grado de internacionalización estimado, no es posible concluir sobre el éxito de la decisión de incursionar en el exterior mediante $M \& A s$, debido a que cada banco tiene comportamientos y estrategias distintas. Por ejemplo, en Bancolombia es clara su estrategia de regionalización de manera paulatina y metódica, pero en los caso de Banco do Brasil y BCP no son constantes y ni representativas con respecto a la medida de los activos, lo que dificulta interpretar si las decisiones de las compras fueron un movimiento estratégico, una oportunidad de negocio o una intención de penetrar en mercados, entre otras.

El grado de internacionalización es una medida particular para cada empresa y expone la situación internacional de la compañía. Es así como se debe tener tacto al comparar los grados de internacionalización entre empresas de diferentes países.

Para el grado de internacionalización es relevante el momento en que se realiza la transacción de $M \& A s$; a medida que el tiempo transcurre, la velocidad de crecimiento del negocio, tanto doméstico como internacional, afecta el indicador.

Se propone que futuros estudios analicen si las $M \& A s$ son determinantes en la evolución del grado de internacionalización, es decir, si se puede encontrar evidencia de que los bancos que han realizado transacciones de $M \& A s$ detentan un mayor grado de internacionalización que aquellos que no han incursionado en estas estrategias de crecimiento.

\section{Referencias}

Asociación Bancaria y de Instituciones Financieras de Colombia -[ASOBANCARIA]. (2103). La internacionalización de la banca y los retos de la supervisión consolidada. Semana Económica, 920. Recuperado de https://cdn2.hubspot.net/hubfs/1756764/Asobancaria $\% 20$ Eventos/ Asobancaria\%20-\%20Semanas-Economicas/SemanasEconomicas-2014-2009/ Sem_920.pdf

Banco do Brasil (s.f.). Sobre nós. Recuperado de http://www.bb.com.br

Bank for International Settlements, BIS (2014). Report of consolidation in the financial sector. Data annex A: patterns in consolidation transactions. Recuperado de http://www.bis.org/publ/gten05annex. pdf

Buch, C. M., \& DeLong, G. L. (2003). Crossborder bank mergers: What lures the rare animal? Journal of Banking \& Finance, 28(9), 2077-2102.

Cardona-Vélez, G. H. (2014). Análisis del impacto de los cambios del control corporativo sobre el valor de las empresas en América Latina. Cuadernos de Economía, 33(62), 163-197. doi: 10.15446/ cuad.econ.v33n62.43671.

Cisneros, C., \& Jiménez, M. (2014). Análisis comparativos de las fusiones y adquisiciones en los planes estratégicos de las empresas mexicanas; capitulo 1: proceso de fusión y adquisición (Trabajo de grado como licenciadas en Contaduría y Finanzas). Universidad de las Américas Puebla, Escuela de Negocios, México. Recuperado de http://catarina.udlap.mx/u_dl_a/ tales/documentos/lcp/cisneros_t_c/capitulo1.pdf 
Comisión Económica para América Latina y el Caribe -[CEPAL]. (2011). La inversión extranjera directa en América Latina $y$ el Caribe: informe 2010 (CEPAL, Informes anuales). Recuperado de http:// www.cepal.org/es/publicaciones/1141-lainversion-extranjera-directa-en-americalatina-y-el-caribe-informe-2010

Cuervo-Cazurra, Á. (2007). Las distancias en el proceso de internacionalización: el caso de Banco Santander. Universia Business Review, 150(especial aniversario Banco Santander), 96-111.

Daniels, J. D., \& Bracker, J. (1989). Profit performance: Do foreign operations make a difference? Management International Review, 29(1), 46-56.

De Nicoló, G., Bartholomew, P., Zaman, J., \& Zephirin M. (2004). Bank consolidation, internationalization and conglomeration: trends and implications for financial risk. Financial Markets, Institutions \& Instruments, 13(4), 173-217.

De Pamphilis, D. (2012). Mergers, acquisitions, and other restructuring activities ( $6^{\mathrm{a}}$ ed.) Nueva York: Academic Press; Elsevier.

Decreto 410 de 1971. Por el cual se expide el Código de Comercio. Diario Oficial No. 33.339. Presidencia de la República, marzo de 1971.

Eppink, D., \& Rhijn, E. D. (1988). The internationalization of Dutch industry. Long Range Planning, 21(5), 54-60.

Estrada, D. (2007). Efectos de las fusiones sobre el mercado financiero colombiano (Banco de la República, Borradores de Economía No 329). Bogotá: Autor. Recuperado de http://www.banrep.gov. co/es/borrador-329
Forsgren, M., \& Johanson, J. (1992). Managing networks in international business. Londres: Routledge.

Grupo Bancolombia(s.f.).Nosotros. Recuperado de http:/www.grupobancolombia.com/ webCorporativa/nosotros/contenido/ historia2.asp

Investing. (2015). Tasa representativa del mercado. Recuperado de www.investing. com

Johanson, J., \& Vahlne, J. E. (1997). The internationalization process of the firm. A model of knowledge development and increasing foreign market commitments. Journal of International Business Studies, $8(1), 23-32$.

Larraín, C. (1995). Internacionalización y supervisión de la banca en Chile. Estudios Públicos, 60, 117-143.

Martínez, M. S., Powell, A., \& VladkovaHollar, I. (2005). Banking on foreigners: the behavior of international bank claims on Latin America, 1985-2000. IMF Staff Papers, 52(3), 430-461.

Mascareña, J. (1999). Introducción a las finanzas corporativas. Madrid: Universidad Complutense de Madrid.

McCann, J. E., \& Gilkey, R. (1990). Fusiones y adquisiciones de empresas. Madrid: Díaz de Santos.

Pessanha, G. R. G., Calegano, C. L. L., Sáfadi, T., \& Ázara, L. N. (2012). Impactos das estrategias de fusão e aquisicão na rentabilidade dos bancos adquirentes: uma aplicação dos modelos de intervenção no setor bancário brasileiro. Revista de Administração Mackenzie, 13(5), 101134. Recuperado de http://repositorio. ufla.br/handle/1/238 
Proméxico (s.f.). ¿Qué es la internacionalización? Recuperado de http://www. promexico.gob.mx/es/mx/definicion-deinternacionalizacion

Ren, S., Eisingerich, A. B., \& Tsai, H.-T. (2014). How do marketing, research and development capabilities, and degree of internationalization synergistically affect the innovation performance of small and medium-sized enterprises (SMEs)? A panel data study of Chinese SMEs. International Business Review, 24(4), 642651. doi: 10.1016/j.ibusrev.2014.11.006

Rodrigues de Paula, L. F. (2003). Los determinantes del reciente ingreso de bancos extranjeros a Brasil. Revista de la CEPAL, 79, 169-188. Recuperado de http://repositorio.cepal.org/handle/11362/10882

Ross, S., Westerfield, R., \& Jaffe, J. (2014). Finanzas corporativas. México: McGrawHill.

Santeiro, M. (2014). Cuestiones básicas y metodología en el proceso de fusiones y adquisiciones (Tesis de Maestría en Banca y Finanzas). Universidade da Coruña, España. Recuperado de http://ruc.udc. es/dspace/bitstream/handle/2183/12458/ SanteiroGarcia_Miguel_TFM_2014.pdf? sequence $=2$

Stopford, J. M., \& Wells, L. T. (1972). Managing the multinational enterprise. Nueva York: Basic Books.
Sullivan, D. (1994). Measuring the degree of internationalization of a firm. Journal of International Business Studies, 25(2), 325-342.

S\&P Capital IQ - McGraw Hill Financial. (2015). Consulta de estados financieros anuales de los bancos (adquiridos y adquirentes) en dólares (Base de datos). Recuperado de https://marketintelligence. spglobal.com/our-thinking/newsroom/sp-global-market-intelligence-a-divisionof-mcgraw-hill-financial-agrees-to-selltwo-assets-to-intercontinental-exchange

Torres, D. (2014). Globalización, empresas multinacionales e historia. Pensamiento y Gestión, 30, 165-185. Recuperado de http://rcientificas.uninorte.edu.co/index. $\mathrm{php} /$ pensamiento/article/view/2254

Thomnson One. (2015). Transacciones de $M \& A s$ (Base de datos). Recuperado de https://www.thomsonone.com/DirectoryServices/2006-04-01/Web.Public/Login. aspx?brandname $=$ www.thomsonone.com $\&$ version $=3.7 .9 .18833 \&$ protocol $=0$

Vernon, R. (1971). Sovereignity at bay: The mtiultiniationtal spread of U.S. enterprises. Nueva York: Basic Books.

Vermeulen, F. (2001). Controlling international expansion. Business Strategy Review, 12(3), 29-36. 
